\title{
APPROXIMATELY CONVEX FUNCTIONS
}

D. H. HYERS AND S. M. ULAM

In previous papers approximately linear functions [1] and approximately isometric transformations $[2 ; 3 ; 4]$ have been studied. ${ }^{1}$ In both cases it was shown that the properties of linearity and isometry are "stable" in a certain sense. For example, it was proved that if a function $f(x)$ satisfies the linear functional equation within an amount $\epsilon$, that is, $|f(x+y)-f(x)-f(y)| \leqq \epsilon$, then there exists an actual solution $g(x)$ of the linear functional equation such that $|g(x)-f(x)| \leqq \epsilon$, where $\epsilon$ is a given positive number.

In the present paper we discuss a similar problem for the property of convexity. We consider real-valued functions defined on subsets of $n$-dimensional Euclidean space $E_{n}$. A function $f(x)$ defined on a convex subset $S$ of $E_{n}$ will be called $\epsilon$-convex if $f(h x+(1-h) y)$ $\leqq h f(x)+(1-h) f(y)+\epsilon$, for all $x$ and $y$ in $S$ and for $0 \leqq h \leqq 1$. Here $\epsilon$ is a fixed positive number. Our object is to show that to an $\epsilon$-convex function $f(x)$ there corresponds a convex function $g(x)$ such that $|f(x)-g(x)| \leqq k \epsilon$, for some constant $k$. In order to prove this we need some results on $\epsilon$-convex functions and on approximating simplices given in the following four lemmas. The paper is self-contained.

Lemma 1. Let $f(x)$ be an $\epsilon$-convex function defined on an n-dimensional simplex $S \subset E_{n}$. Let the vertices of the simplex be $p_{0}, p_{1}, \cdots, p_{n}$, then if $x=\sum_{i=0}^{n} \alpha_{i} p_{i}, \alpha_{i}>0, \sum_{i=0}^{n} \alpha_{i}=1$ is any point of $S$, we have

$$
f(x) \leqq \sum_{i=0}^{n} \alpha_{i} f\left(p_{i}\right)+2 k_{n} \epsilon \text {, }
$$

where $k_{n}=\left(n^{2}+3 n\right) /(4 n+4)$.

Proof. We prove the inequality by induction on $n$. For $n=1$, (1) reduces to the statement of $\epsilon$-convexity, so it is true for $n=1$. We assume that (1) holds for $n$ replaced by $n-1$, and prove it for $n$ dimensions. The case in which some $\alpha_{i}=1$ is trivial, for in this case $x=p_{i}$, so we may assume that $\alpha_{i}<1$ for $i=1, \cdots, n+1$. For convenience we may also assume that $\alpha_{n} \geqq \alpha_{j}, j=0, \cdots, n-1$. Put $h=1-\alpha_{n}, a_{j}=\alpha_{j} / h, j=0, \cdots, n-1$, and $q=\sum_{j=0}^{n-1} a_{j} p_{j}$. Then $x$ $=\sum_{i=0}^{n} \alpha_{i} p_{i}=h q+(1-h) p_{n}$, and since $f$ is $\epsilon$-convex,

$$
f(x) \leqq h f(q)+(1-h) f\left(p_{n}\right)+\epsilon .
$$

Presented to the Society, April 28, 1951; received by the editors February 6, 1952.

1 For a discussion of these and other related questions, see [6]. 
By the induction hypothesis,

$$
f(q) \leqq \sum_{j=0}^{n-1} a_{j} f\left(p_{j}\right)+\frac{(n-1)(n+2)}{2 n} \epsilon .
$$

Substituting (3) into (2), we get

$$
f(x) \leqq \sum_{i=0}^{n} \alpha_{i} f\left(p_{i}\right)+\left\{1+\frac{h(n-1)(n+2)}{2 n}\right\} \epsilon .
$$

Since $\alpha_{n} \geqq \alpha_{j}, j=0, \cdots, n-1$, the minimum value which $\alpha_{n}$ can have is $1 /(n+1)$, so the maximum value which $h$ can have is 1 $-1 /(n+1)=n /(n+1)$. Consequently an upper bound for the expression in brackets in inequality (4) is

$$
1+\frac{(n-1)(n+2)}{2(n+1)}=\frac{n^{2}+3 n}{2 n+2} .
$$

Thus the lemma has been established.

LEMMA 2. Let $f(x)$ be an $\epsilon$-convex function defined on an open convex set $G \subset E_{n}$. Then on each closed bounded subset $B$ of $G, f(x)$ is bounded.

Proof. $f$ is bounded from above, since $B$ may be covered with a finite number of $n$-dimensional simplices, each contained in $G$, and $f$ is bounded on each simplex by Lemma 1 .

To prove that $f$ is bounded from below on $B$, let $B$ be covered with a finite number of closed spheres $S_{i}$, such that each $S_{i}$ is contained in $G$. Let $x_{i}$ be the center of $S_{i}$, and let $x_{i}+y$ be any point of the sphere $S_{i}$. Then by $\epsilon$-convexity

or

$$
f\left(x_{i}\right) \leqq 2^{-1} f\left(x_{i}+y\right)+2^{-1} f\left(x_{i}-y\right)+\epsilon,
$$

$$
f\left(x_{i}+y\right) \geqq 2 f\left(x_{i}\right)-f\left(x_{i}-y\right)-2 \epsilon .
$$

Now $x_{i}-y$ belongs to the sphere $S_{i}$, and since $S_{i}$ is a closed subset of $G, f\left(x_{i}-y\right)$ is bounded from above as $x_{i}+y$ varies over $S_{i}$. Hence $f\left(x_{i}+y\right)$ is bounded from below for $x_{i}+y \in S_{i}$, and it follows that $f$ is bounded from below on $B$. The proof of the following two lemmas is left to the reader.

LеммA 3. Let $x$ lie in an $n$-dimensional simplex with vertices $q_{0}, q_{1}, \cdots, q_{n}$, so that $x=\sum_{i=0}^{n} \alpha_{i} q_{i}, \alpha_{i} \geqq 0$, and $\sum_{i=0}^{n} \alpha_{i}=1$. Suppose also that we have $n+1$ sequences $\left\{q_{i}^{(\nu)}\right\}(i=0, \cdots, n ; \nu=1,2,3, \cdots)$ of points such that $q_{i}^{(\nu)} \rightarrow q_{i}$ as $\nu \rightarrow \infty$, and that for each $\nu, x$ also lies in the $n$-dimensional simplex with vertices $q_{i}^{(v)}$ so that 


$$
x=\sum_{i=0}^{n} \alpha_{i}^{(p)} q_{i}^{(p)}
$$

where $\alpha_{i}^{(\nu)} \geqq 0$ and $\sum_{i=0}^{n} \alpha_{i}^{(\nu)}=1$. Then as $\nu \rightarrow \infty, \alpha_{i}^{(\nu)} \rightarrow \alpha_{i}$.

Lemma 4. Suppose $x$ is interior to an n-dimensional simplex in $E_{n}$ whose vertices are $q_{0}, q_{1}, \cdots, q_{n}$. Then if $q_{i}^{(\nu)} \rightarrow q_{i}$ in $E_{n}$ as $\nu \rightarrow \infty$. $(i=0, \cdots, n), x$ is also interior to the simplex $S_{n}^{(\nu)}$ whose vertices are $q_{i}^{(\nu)}(i=0, \cdots, n)$, for sufficiently large $n$.

THEOREM 1. Let $f(x)$ be $\epsilon$-convex on an open convex set $G \subset E_{n}$, and let $B$ be any closed bounded convex subset of $G$. Then there exists a convex function $\phi(x)$ on $B$ such that

$$
|\phi(x)-f(x)| \leqq k_{n} \epsilon, \quad \text { for } x \in B,
$$

where $k_{n}=\left(n^{2}+3 n\right) /(4 n+4)$.

Proof. Let $H$ be a bounded convex open subset of $G$ such that $B \subset H$, and $\bar{H} \subset G$. Since $B$ is a compact subset of the open convex set $G$, the existence of such an $H$ is easily shown. Let $K$ denote the convex hull of the closure of the graph of the function $f(x)$ for $x \in \bar{H}$, so that $K$ is a convex set in $E_{n+1}$.

Define, for $x=\left(x_{1}, \cdots, x_{n}\right) \in \bar{H}, g(x)=\inf \left[y ;\left(x_{1}, x_{2}, \cdots, x_{n}, y\right)\right.$ $\in K]$. Since $f(x)$ is bounded on $\bar{H}$ by Lemma $2, K$ is a compact set in $E_{n+1}$ and $g(x)$ is well defined on $\bar{H}$. It is easily seen that $g(x)$ is a convex function, and that $g(x) \leqq f(x)$ for $x \in H$. Given a point $x \in B$, let $p$ denote the point $\left(x_{1}, x_{2}, \cdots, x_{n}, g(x)\right)$ in $E_{n+1}$. Now $p$ evidently belongs to the boundary of $K$, and since $K$ is closed, it also belongs to $K$. By a well known theorem, ${ }^{2} p$ lies on an $m$-dimensional simplex $S_{m}$ whose vertices are points or limit points of the graph of $f(x)$ for $x \in \bar{H}$, where $m \leqq n+1$. Notice that the assertion is actually true for some $m \leqq n$, for if $p$ were in the interior of an $(n+1)$-dimensional simplex with vertices in $K$, then $p$ would lie in the interior of $K$ and not on its boundary.

There are three possible cases.

(i) $p$ is a point of the graph of $f$.

(ii) $p$ is a limit point of the graph of $f$.

(iii) $p$ is "interior" 3 to some simplex $S$ whose dimension is positive and less than or equal to $m$, and whose vertices are points or limit points of the graph of $f$.

In case (i), $f(x)=g(x)$, and there is nothing to prove. In case (ii)

2 See $[5$, p. 9].

"A point will be called "interior" to a simplex $S$ of dimension $r$ if it belongs to $S$ but not to any face of lower dimension than $r$. 
it is convenient to translate the axes so that the origin of coordinates lies at the point $x$ so that $x=0$. Then by hypothesis there exists a sequence of distinct points $x^{(\mu)} \in H \subset E_{n}$ tending to zero such that $\lim _{\mu \rightarrow \infty} f\left(x^{(\mu)}\right)=g(0)$. It is clear that an infinite number of these points must all lie in some one of the $2^{n}$-tants determined by the coordinate hyperplanes. For definiteness, let us assume the first $2^{n}$-tant contains an infinite number of these points. We denote them by $x^{(v)}$, so that all the coordinates of each $x^{(\nu)}$ may be assumed to be non-negative. Now choose on each coordinate axis a point $p_{j}$ whose $j$ th coordinate is negative, the others being zero, $j=1,2, \cdots, n$, such that $p_{j} \in H$. Consider the simplex $S^{(v)}$ whose vertices are $p_{1}, p_{2}, \cdots, p_{n}$ and $x^{(v)}$. Then the origin belongs to this simplex, and there exist $\alpha_{i}^{(p)}, i=1, \cdots, n+1$, such that

$$
\sum_{j=1}^{n} \alpha_{j}^{(v)} p_{j}+\alpha_{n+1}^{(p)} x^{(v)}=0,
$$

where $\alpha_{j}^{(\nu)} \geqq 0, \alpha_{n+1}^{(\nu)}>0$, and $\sum_{i=1}^{n+1} \alpha_{i}^{(\nu)}=1$.

To prove this, let $p_{j j}$ be the $j$ th coordinate of the point $p_{j}$ and let $x_{j}^{(\nu)}$ be the $j$ th coordinate of the point $x^{(v)}$. Then the "vector" equation (5) may be written in the form:

$$
\alpha_{j}^{(v)} p_{j j}+\alpha_{n+1}^{(v)} x_{j}^{(v)}=0, \quad j=1, \cdots, n,
$$

where $p_{j j}<0$, and $x_{j}^{(\nu)} \geqq 0$. Since $x^{(\nu)} \neq 0$, at least one of the $x_{j}^{(\nu)}$ must be positive. If $x_{j}^{(\nu)}=0$, choose $\alpha_{j}^{(\nu)}=\rho_{j}^{(\nu)}=0$. If $x_{j}^{(\nu)} \neq 0$ equation (6) determines the ratio $\rho_{j}^{(\nu)}=\alpha_{j}^{(\nu)} / \alpha_{n+1}^{(\nu)}$, which in this case is evidently positive. The value of $\alpha_{n+1}^{(\nu)}$ is then determined by the requirement that $\sum_{i=1}^{n+1} \alpha_{i}^{(\nu)}=\left(1+\sum_{j=1}^{n} \rho_{j}^{(\nu)}\right) \alpha_{n+1}^{(v)}=1$. Thus relation (6) is established. By Lemma 1, it follows that

$$
f(0) \leqq \sum_{j=1}^{n} \alpha_{j}^{(p)} f\left(p_{i}\right)+\alpha_{n+1}^{(v)} f\left(x^{(p)}\right)+2 k_{n} \epsilon
$$

Now as $\nu \rightarrow \infty, x^{(p)} \rightarrow 0$. Hence by (6), $\alpha_{j}^{(\nu)} \rightarrow 0$ for $j=1, \cdots, n$. It follows that $\alpha_{n+1}^{(\nu)} \rightarrow 1$. Since $f\left(x^{(v)}\right) \rightarrow g(0)$, we have $f(0) \leqq g(0)+2 k_{n} \epsilon$, or $f(x) \leqq g(x)+2 k_{n} \epsilon$.

We now turn to case (iii). Here $p$ lies in the interior of an $r$-dimensional simplex $S_{r}(1 \leqq r \leqq n)$ whose vertices $p_{i}(i=0,1, \cdots, r)$ are points or limit points of the graph of $f$.

Let $\pi$ be a supporting hyperplane of $K \subset E_{n+1}$ through the point $p$. Now $p$ is interior to at least one line segment $S_{1}$ belonging to $S_{r}$ and hence to $K$. Any such line segment $S_{1}$ must lie in the hyperplane $\pi$, for otherwise $S_{1}$ would pierce the hyperplane $\pi$ at $p$ so that part of 
$S_{1}$ would lie on one side of $\pi$ and part on the other, which is impossible since all of $K$ lies on one side of $\pi$. It follows that $S_{r}$, and hence its vertices $p_{i}$, lies in $\pi$, and the $p_{i}$ are boundary points of $K$.

This supporting hyperplane $\pi$ cannot be perpendicular to $E_{n}$, for in this case $\pi$ would project (orthogonally) into a hyperplane in $E_{n}$ which would be a supporting hyperplane of the projection of the convex set $K$ and which would contain the point $x$. Thus $x$ would be on the boundary of the projection of $K$. But the projection of $K$ includes the open set $H$ which by hypothesis contains $x$, so $x$ cannot lie on the boundary of $K$ 's projection, and we have a contradiction.

Therefore the projection of $S_{r}$ onto $E_{n}$ is a simplex $\Sigma_{r}$ of the same dimension $r$, and the interior of $S_{r}$ projects into the interior of $\Sigma_{r}$, so that the point $x$ which is the projection of $p$ lies in the interior of $\Sigma_{r}$.

We use double subscripts to denote the coordinates of the vertices $p_{i}$ of $S_{r}$, and we denote the projections of these vertices onto $E_{n}$ by $q_{0}, q_{1}, \cdots, q_{r}$. Then by hypothesis there exist sequences $q_{i}^{(\nu)}$ such that $p_{i, n+1}=\lim _{\nu \rightarrow \infty} f\left(q_{i}^{(\nu)}\right)$, where $\lim _{\nu \rightarrow \infty} q_{i}^{(\nu)}=q_{i}$, and $q_{0}, \cdots, q_{r}$ are the vertices of the $r$-dimensional simplex $\Sigma_{r} \subset E_{n}$, which contains the point $x$ in its interior. Our object is to construct a simplex $S_{n}^{(\nu)}$ of dimension $n$ in $E_{n}$ such that $x$ is interior to $S_{n}^{(v)}$, and such that $r$ of its vertices are points $q_{0}^{(\nu)}, \cdots, q_{r}^{(\nu)}$. We can then apply Lemma 1 to this simplex and take the limit in the resulting inequality as $\nu \rightarrow \infty$.

Suppose first that $r=n$. In this case, $x$ is interior to the $n$-dimensional simplex $\Sigma_{n} \subset E_{n}$, so that

$$
x=\sum_{i=0}^{n} \alpha_{i} q_{i}, \quad \alpha_{i}>0, \quad \sum_{i=0}^{n} \alpha_{i}=1 .
$$

Since $g_{i}^{(\nu)} \rightarrow q_{i}$ in $E_{n}$ as $\nu \rightarrow \infty$, it follows by Lemma 4 that $x$ $=\sum_{i=0}^{n} \alpha_{i}^{(\nu)} q_{i}^{(\nu)}, \alpha_{i}^{(\nu)}>0, \sum_{i=0}^{n} \alpha_{i}^{(\nu)}=1$. Hence by Lemma $3, \alpha_{i}^{(\nu)} \rightarrow \alpha_{i}$ as $\nu \rightarrow \infty$.

Now by Lemma 1 , we have $f(x) \leqq \sum_{i=0}^{n} \alpha_{i}^{(\nu)} f\left(q_{i}^{(\nu)}\right)+2 k_{n} \epsilon$. By taking limits as $\nu \rightarrow \infty$ we get

$$
f(x) \leqq \sum_{i=0}^{n} \alpha_{i} p_{i, n+1}+2 k_{n} \epsilon=g(x)+2 k_{n} \epsilon .
$$

Now let us suppose that $1 \leqq r \leqq n$. Let $F_{r}$ be the $r$-dimensional flat containing $\Sigma_{r}$. Now if for all but a finite number of $\nu$ 's, the $q_{i}^{(\nu)}$, $i=0, \cdots, n ; \nu=1,2,3, \cdots$, are contained in $F_{r}$, then $q_{i}^{(\nu)} \rightarrow q_{i}$ in $F_{r}$ and one has essentially case (iiia) with $r$ replacing $n$, so the proof follows as before. 
Next suppose that an infinity of points $q_{i}^{(\nu)}$ for some $i$ lie outside this flat. We may as well assume (by relabeling and suppressing a subsequence if necessary) that all of the $q_{0}^{(\nu)}$ lie outside $F_{r}$.

Let us choose a new coordinate system with origin at $q_{0}$ and with the first $r$ axes belonging to $F_{r}$, so that the equations of $F_{r}$ are $z_{j}=0, j=r+1, \cdots, n$. The last $n-r$ coordinates $q_{0, r+1}^{(\nu)}, \cdots, q_{0, n}^{(\nu)}$ of the point $q_{0}^{(\nu)}$ cannot all be zero for any $\nu$. It follows that for some fixed $j, q_{0, j}^{(\nu)} \neq 0$, for all $\nu$. We may without loss of generality assume that $q_{0, r+1}^{(\nu)} \neq 0$, for all $\nu$. Now there must be an infinity of the numbers $q_{0, r+1}^{(p)}$ which are either all positive or all negative, and by reversing the $(r+1)$ st coordinate axis if necessary, we may assume that $q_{0, r+1}^{(\nu)}>0$ for all $\nu$.

Next, if $r+1<n$, we consider $q_{0, r+2}^{(v)}$. If $q_{0, r+2}^{(v)}=0$ for all but a finite number of $\nu$ 's, we rotate the $z_{r+1}$ and $z_{r+2}$ axes through an acute angle, keeping all of the other axes fixed, in such a way that after the rotation $q_{0, r+1}^{(p)}$ will still be positive and $q_{0, r+2}^{(\nu)}$ will become positive for all but a finite number of $\nu$ 's.

On the other hand if $q_{0, r+2}^{(\nu)} \neq 0$ for an infinite number of $\nu$ 's, then for an infinite number of $\nu$ 's, these numbers are all positive or all negative. By reversing the $z_{r+2}$-axis if necessary we have $q_{0, r+2}^{(\nu)}>0$ for an infinite number of $\nu$ 's. Thus by suppressing a subsequence if necessary we can arrange matters so that $q_{0, r+1}^{(\nu)}>0$ and $q_{0, r+2}^{(\nu)}>0$ for all $\nu$.

If $r+2<n$, we proceed in the same way, with $r+1$ replacing $r$, and so on. Thus, there will exist a coordinate system in $E_{n}$ and sequences of points $q_{i}^{(p)} \rightarrow q_{i}(i=0,1, \cdots, r)$ such that the origin lies at the point $q_{0}$, and $q_{i, j}=0, q_{0, j}^{(\nu)}>0$ for $j=r+1, \cdots, n$, where $f\left(q_{i}^{(p)}\right) \rightarrow p_{i, n+1}, x=\sum_{i=0}^{r} \alpha_{i} q_{i}, g(x)=\sum_{i=0}^{r} \alpha_{i} p_{i, n+1}, \sum_{i=0}^{r} \alpha_{i}=1, \alpha_{i}>0$.

Now let $q_{i}(i=r+1, \cdots, n)$ be a point in $H$ whose $(r+1)$ st coordinate is a negative number and whose other coordinates are all zero. We now show that $x$ is interior to the $n$-dimensional simplex whose vertices are $q_{0}^{(\nu)}, q_{1}, q_{2}, \cdots, q_{n}$, for sufficiently large $\nu$.

Thus we must show the existence of positive numbers $\beta_{i}$ $(i=0, \cdots, n)$ with $\sum_{i=0}^{n} \beta_{i}=1$ such that $x=\sum_{i=0}^{r} \quad \alpha_{i} q_{i}=\beta_{0} q_{0}^{(\nu)}$ $+\sum_{i=0}^{n} \beta_{i} q_{i}$. That is, the $\beta_{i}$ are to satisfy the following system of $n+1$ linear equations:

$$
\begin{aligned}
\beta_{0} q_{0, j}^{(v)}+\sum_{i=0}^{r} \beta_{i} q_{i, j} & =\sum_{i=0}^{r} \alpha_{i} q_{i, j} \quad(j=1, \cdots, r), \\
\beta_{0} q_{0, j}^{(\nu)}+\beta_{j} q_{j, j} & =0 \quad(j=r+1, \cdots, n), \\
\sum_{i=0}^{n+1} \beta_{i} & =1 .
\end{aligned}
$$


Since $\alpha_{i}>0, \quad \sum_{i=0}^{r} \alpha_{i}=1$, and $q_{0, j}^{(\nu)} \rightarrow q_{0, j}=0$, it follows that for $0<\beta_{0}<1$ there will exist a $\nu_{0}$, independent of $\beta_{0}$, such that the first $r$ equations of the system (8) have solutions for $\beta_{i}, i=1, \cdots, r$, which are between zero and one, whenever $\nu \geqq \nu_{0}$. Since $q_{j, j}$ and $q_{0, j}^{(\nu)}$ are of opposite signs by construction for $j=r+1, \cdots, n$, it is clear that the next $n-r$ equations will also have solutions $\beta_{j}, j=r$ $+1, \cdots, n$, which are between zero and one when $\beta_{0}$ is, and when $\nu$ is sufficiently large. With the help of the last equation all the $\beta$ 's may be determined, with $0<\beta_{i}<1, i=0, \cdots, n$.

Next, for a given $\nu$, so large that $x$ is interior to the simplex with vertices $q_{0}^{\nu}, q_{1}, \cdots, q_{n}$, there will exist by Lemma 4 an index $\mu=\mu(\nu)$ such that $x$ is also interior to the simplex with vertices $q_{0}^{\nu}, q_{1}^{\mu}, q_{2}^{\mu}, \cdots$, $q_{r}^{\mu}, q_{r+1}, \cdots, q_{n}$. Let one such index $\mu$ be determined for each $\nu$ and put $\bar{q}_{i}^{\nu}=q_{i}^{\mu(\nu)}, i=1, \cdots, r$. For convenience we also put $\bar{q}_{0}^{\nu}=q_{0}^{\nu}$. Then there exist $\alpha_{i}>0, i=0,1, \cdots, n$, such that $\sum_{i=0}^{n} \alpha_{i}^{p}=1$ and $x$ $=\sum_{i=0}^{r} \alpha_{i} q_{i}=\sum_{i=0}^{r} \alpha_{i}^{i} \bar{q}_{i}+\sum_{i=r+1}^{n} \alpha_{i} q_{i}$. By Lemma 1 we have

$$
f(x) \leqq \sum_{i=0}^{r} \alpha_{i} f\left(\bar{q}_{i}\right)+\sum_{j=r+1}^{n} \alpha_{j} f\left(q_{i}\right)+2 k_{n} \epsilon .
$$

Now as $\nu \rightarrow \infty, \bar{q}_{i}^{y} \rightarrow q_{i}, f\left(\bar{q}_{i}^{p}\right) \rightarrow p_{i, n+1}$, and, by Lemma 3, we know that $\alpha_{i}^{\nu} \rightarrow \alpha_{i}$ for $i=0,1, \cdots, r$, while $\alpha_{j}^{\prime} \rightarrow 0, j=r+1, \cdots, n$. Hence by letting $\nu \rightarrow \infty$ in the last inequality we get

$$
f(x) \leqq \sum_{i=0}^{r} \alpha_{i} p_{i, n+1}+2 k_{n} \epsilon=g(x)+2 k_{n} \epsilon .
$$

We have proved that for any point $x \in B, g(x) \leqq f(x) \leqq g(x)+2 k_{n} \epsilon$, where $g(x)$ is a convex function. Now define $\phi(x)=g(x)+k_{n} \epsilon$. Then $\phi(x)$ is convex and

$$
|\phi(x)-f(x)| \leqq k_{n} \epsilon \quad \text { for } x \in B .
$$

This completes the proof of theorem 1 .

TheOREM 2. If $f(x)$ is an $\epsilon$-convex function defined on a convex open subset of $G$ of $E_{n}$, then there exists a convex function $\phi(x)$ defined on $G$ such that $|f(x)-\phi(x)| \leqq k_{n} \epsilon$.

Proof. Let $H_{v}, \nu=1,2,3, \cdots$, be a sequence of convex, compact subsets of $G$ such that $H_{\nu+1} \subset H_{\nu}$ and such that $G=\bigcup_{\nu-1}^{\infty} H_{\nu}$ (the existence of such a sequence is easily demonstrated). Then by Theorem 1 , there exists for each $\nu$ a convex function $\phi_{\nu}(x)$ on $H$, such that $\left|\phi_{\nu}(x)-f(x)\right| \leqq k_{n} \epsilon$, for $x \in H_{\nu}$. For each fixed positive integer $\mu$, the function $f(x)$ is bounded on $H_{\mu}$ by Lemma 2 . Hence the sequence $\left\{\phi_{\nu}(x)\right\}$ is defined and uniformly bounded on $H_{\mu}$ for $\nu \geqq \mu$. By a well 
known selection theorem there exists a subsequence $\left\{\phi_{1 p}(x)\right\}$ of the $\phi_{\nu}(x)$ which converges for all $x \in H_{1}$. Similarly there is a subsequence $\left\{\phi_{2 p}(x)\right\}$ of the $\phi_{1 p}(x)$ which is defined and convergent on $H_{2}$, and so on. Now consider the sequence $\left\{\phi_{p p}(x)\right\}, p=1,2,3, \cdots$. For any given $x \in G$, there exists a positive integer $m$ so that $x \in H_{m}$. Hence for $p \geqq m$, the sequence $\left\{\phi_{p p}(x)\right\}$ is defined and converges to a limit $\phi(x)$. Thus $g(x)$ is defined, is convex, and satisfies the inequality $|\phi(x)-f(x)| \leqq k_{n} \in$ for $x \in G$.

\section{REFERENCES}

1. D. H. Hyers, On the stability of the linear functional equation, Proc. Nat. Acad. Sci. U.S.A. vol. 27 (1941) pp. 222-224.

2. D. H. Hyers and S. M. Ulam, On approximate isometries, Bull. Amer. Math. Soc. vol. 51 (1945) pp. 288-292.

3. - Approximate isometries of the space of continuous functions, Ann. of Math. vol. 48 (1947) pp. 285-289.

4. D. G. Bourgin, Approximate isometries, Bull. Amer. Math. Soc. vol. 52 (1946) pp. 704-714.

5. T. Bonnesen and W. Fenchel, Konvexe Körper, New York, 1948.

6. D. G. Bourgin, Classes of transformations and bordering transformations, Bull. Amer. Math. Soc. vol. 57 (1951) pp. 223-237.

The University of Southern California and

The Los Alamos Scientific Laboratory 